\title{
Prostate cancer: diagnostic criteria and role of immunohistochemistry
}

\author{
Cristina Magi-Galluzzi ${ }^{1,2}$ \\ ${ }^{1}$ Department of Pathology, Cleveland Clinic Lerner College of Medicine, Cleveland, OH, USA and ${ }^{2}$ R.T. \\ Pathology and Laboratory Medicine Institute, Cleveland Clinic, Cleveland, OH, USA
}

\begin{abstract}
The diagnosis of prostate cancer is based on microscopic criteria. Presently, prostate needle biopsy interpretation can be a challenge for the pathologist due to the increased number of specimens with limited amount of suspicious glands and minimal atypia. It is critical for the pathologist to have an organized methodical approach when considering the morphological features enabling a definitive diagnosis of prostate cancer. Although several diagnostic criteria and supportive features have been advocated, only few findings are absolutely specific and diagnostic of prostate cancer. The diagnosis of prostate cancer relies on a combination of architectural and cytological features that are reviewed in detail herein. Infiltrative growth pattern, prominent nucleoli and lack of basal cells are the most useful diagnostic criteria. Perineural invasion, glomerulation and mucinous fibroplasia are pathognomonic features of prostate cancer, although uncommon on small prostate cancer foci. The role of immunohistochemistry in establishing a diagnosis of limited prostate is addressed. Modern Pathology (2018) 31, S12-S21; doi:10.1038/modpathol.2017.139
\end{abstract}

Prostate needle biopsy is the gold standard to establish a diagnosis of prostatic adenocarcinoma. PSA screening, digital rectal examination, a higher number of radiologically visible lesions detected by transrectal ultrasound and the use of thin biopsy needle (18-gauge), allowing to perform more biopsies with less morbidity, have all resulted in an increase in the number of prostate biopsies. The current challenge for the pathologist is the difficulty in prostate needle biopsy interpretation due to the raise of specimens with limited amount of suspicious glands and minimal atypism.

The diagnosis of prostate cancer is based on microscopic criteria. Clinical history, although important, should not influence the histological interpretation of prostate needle core biopsy specimens. It is critical for the pathologist to have an organized methodical approach when considering the morphological features enabling the distinction between prostate cancer, benign mimickers and atypical small acinar proliferation, suspicious but not diagnostic of prostate cancer.

The first important step, before searching for diagnostic findings and supporting features, is to scan needle biopsy sections at low- and high-power magnification, to appreciate the architectural and cytological features of benign glands present in the tissue. As variability in

Correspondence: Professor C Magi-Galluzzi, MD, PhD, R. T. Pathology and Laboratory Medicine Institute, Cleveland Clinic, 9500 Euclid Avenue, L25, Cleveland, OH 44195, USA.

E-mail: magic@ccf.org

Received 3 August 2017; accepted 4 August 2017 biopsy fixation, processing, section thickness and $\mathrm{H} \& \mathrm{E}$ staining influence nuclear size, recognition of nucleoli, chromasia and cytoplasmic staining, benign glands serve as reference when evaluating atypical glands. ${ }^{1}$ When assessing small foci of atypical glands on needle biopsy specimens, the pathologist should pay attention to differences, even subtle, between benign glands and atypical glands in terms of nuclear features, cytoplasmic features and intraluminal content. ${ }^{2}$

It is helpful to mentally list all the features favoring a diagnosis of prostate cancer on one side of an imaginary balance sheet and all the features favoring a benign diagnosis on the opposite side. Although several diagnostic criteria and supportive features have been advocated, only few findings are absolutely specific and diagnostic of prostate cancer, as most may be also present in benign mimickers. Accordingly, the diagnosis of prostate cancer relies on a combination of architectural and cytological features ${ }^{3,4}$ after excluding potential benign mimickers. In cases where several features favor a diagnosis of carcinoma, yet others favor a benign diagnosis, the lesion should be considered suspicious but not diagnostic for cancer. In such cases, a repeat biopsy should be recommended if clinically indicated.

\section{Criteria to establish a diagnosis of prostate cancer}

Infiltrative growth pattern, absence of basal cells and nuclear atypia, in the form of nuclear enlargement 
and prominent nucleoli, are considered major criteria to establish a diagnosis of prostate cancer. ${ }^{1,5}$ However, no single major diagnostic criterion is by itself diagnostic of malignancy.

Minor or 'soft' diagnostic criteria are found in a smaller proportion of cases and are not as influential as major criteria; however, the diagnosis of prostate cancer can still be rendered in the presence of multiple minor criteria in the absence of major criteria. ${ }^{6}$

Although the number of malignant glands required to make a diagnosis of prostate cancer varies depending on circumstances, ${ }^{4,5}$ many urological pathologists are comfortable rendering a diagnosis of prostatic adenocarcinoma in the presence of three or more glands satisfying architectural and cytological diagnostic criteria; nevertheless, there is no absolute number of glands below which prostatic carcinoma should not be diagnosed., ${ }^{1,6}$ The general consensus is that the number of glands required to make a definitive diagnosis of prostate cancer is inversely proportional to the number of criteria present.

\section{Architectural features}

Architectural features are best evaluated at low-tomedium magnification. The infiltrative growth pattern is a major criteria in diagnosing prostate cancer. The epithelial-stromal relationship and the spatial arrangement of prostatic acini are very useful tools when assessing prostate biopsy specimens. In contrast to benign glands, the spacing between malignant acini is very variable. Although it may be difficult to appreciate an infiltrative growth pattern in narrow needle biopsies with a limited number of atypical glands, when present it is a highly reliable feature in establishing a malignant diagnosis. ${ }^{4,5}$ The infiltrative growth pattern frequently presents as small atypical glands randomly scattered between larger, more complex and often paler benign glands (Figure 1). ${ }^{1}$ Benign glands have soft contour, papillary infolding and branching in contrast to the typically sharp luminal contour of small malignant glands and acini (Figure 1).

The haphazard arrangement of clusters or single atypical glands dispersed within the prostatic stroma adjacent to benign glands is indicative of invasion. Another low-magnification pattern of infiltrative growth that should raise concern for malignancy is the presence of crowded glands in a linear arrangement, spanning the width of the biopsy core (Figure 2); uncommon patterns of invasion distinctive of higher grade prostate cancer are cords of cells (Figure 3a), single cells and cribriform glands (Figure 3b). ${ }^{4}$ Typically, invasive prostate glands do not elicit a desmoplastic or inflammatory response. ${ }^{1}$

Infiltrative growth pattern has been reported in $79-100 \%$ of minimal prostatic adenocarcinoma cases. ${ }^{5,7,8}$ When an infiltrative growth pattern is not
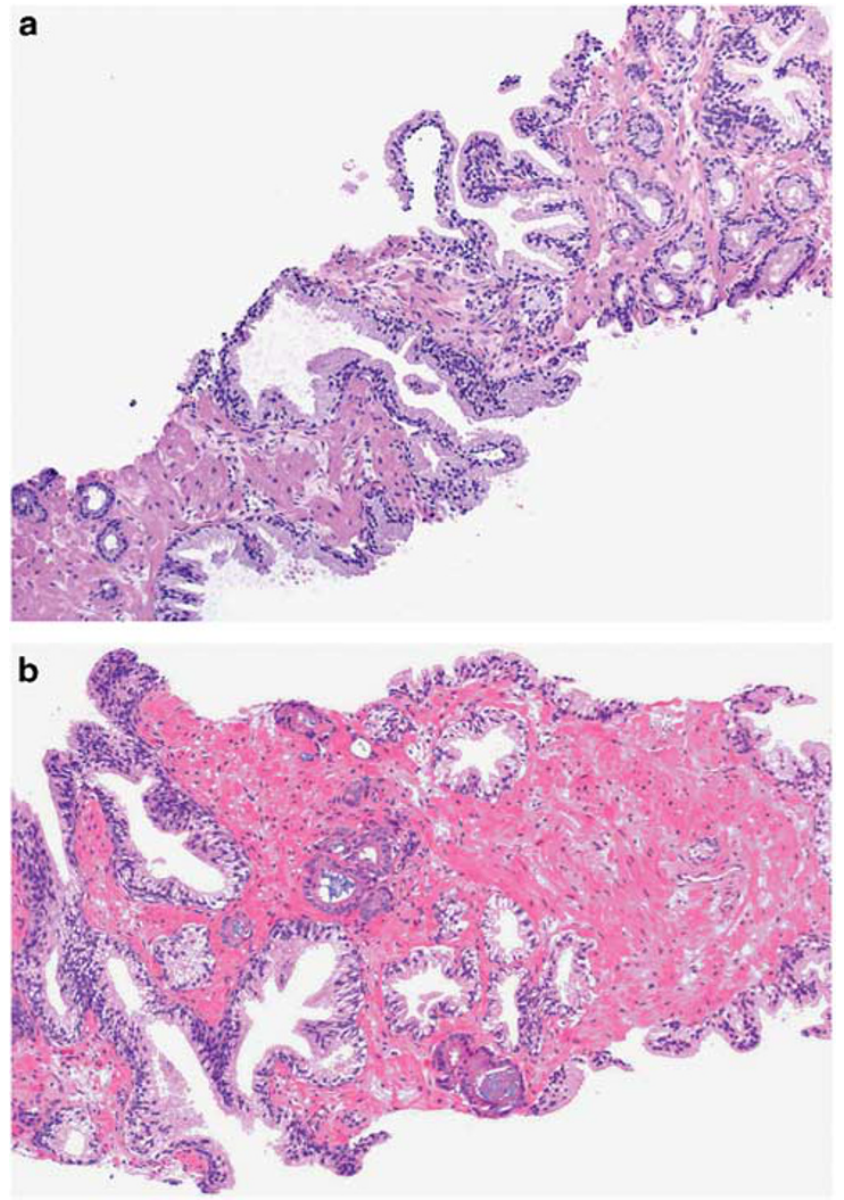

Figure 1 (a and b) Infiltrative growth pattern of small atypical glands randomly scattered between larger, more complex and often paler benign glands. The spacing between malignant acini is very variable. The disorderly arrangement is indicative of stromal invasion. The benign glands have soft contour, papillary infolding and branching in contrast to the sharp luminal contour of the small malignant acini.

evident, the presence of closely packed small acini with size variation, nuclear atypia and lack of basal cells are helpful features in establishing a malignant diagnosis.

\section{Nuclear features}

Nuclear atypia is a major diagnostic criterion in establishing a diagnosis of prostate cancer and is best surveyed at high magnification. ${ }^{1,3,5}$ Nuclear atypia often manifests in the form of nuclear and nucleolar enlargement (Figure 4). Nuclear hyperchromatism may also help in distinguishing malignant from benign glands $\mathrm{s}^{2,7}$ (Figure 4b).

Prominent nucleoli (Figure 5) are present in the majority of malignant cells and have been considered one of the most important features for the diagnosis of prostate cancer. ${ }^{9-13}$ The definition of nucleolar prominence varies from $>1$ to $>3 \mu \mathrm{m} \cdot{ }^{9,14}$ Although not present in all cases, prominent 

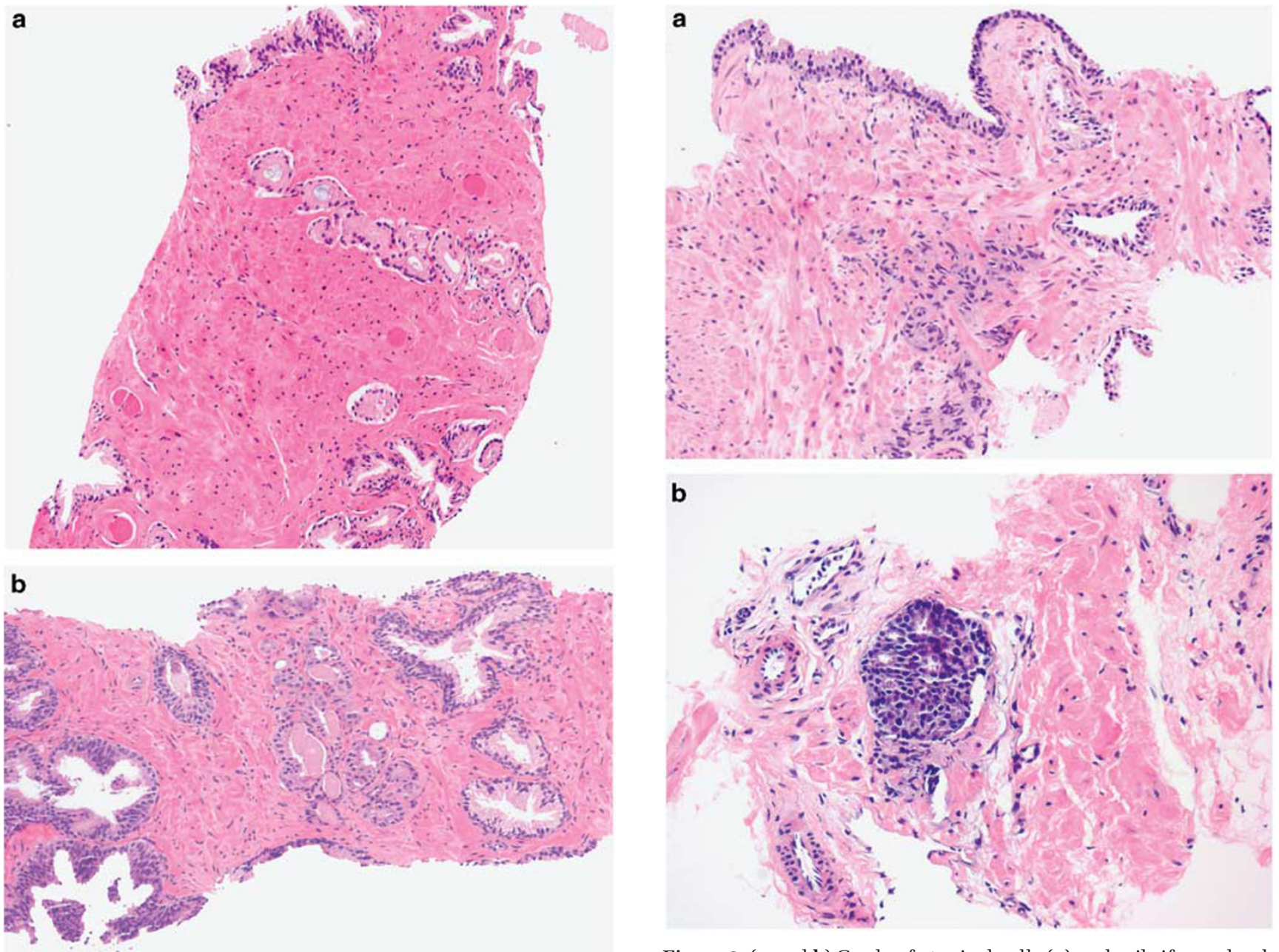

Figure 2 (a and b) Linear arrangement of crowded small-tointermediate atypical glands, spanning the width of the biopsy core. This pattern of growth seen at low magnification is suspicious of malignancy.

nucleoli have been detected to some extent in 76$100 \%$ of prostate cancer cases. ${ }^{4,5,7,8,15}$ In the prostate biopsies series reviewed by Epstein, ${ }^{4}$ prominent nucleoli was the most common finding when only one or two diagnostic criteria were present.

The identification of two or more nucleoli, particularly when eccentrically located, is virtually diagnostic of malignancy; ${ }^{16}$ however, this finding has limited application as many prostate cancers have centrally located nucleoli similar to benign glands. ${ }^{6}$

It is important to keep in mind that some unusual variants of prostate cancer, such as foamy carcinoma, are characterized by bland nuclear features, without prominent nucleoli ${ }^{17}$ and that the diagnosis of highgrade prostatic intraepithelial neoplasia rests in many cases on the detection of prominent nucleoli. ${ }^{18,19}$ Conversely, inflamed benign glands, atrophy and atypical basal cell hyperplasia can occasionally display prominent nucleoli. ${ }^{20}$ Varma et $a 1^{15}$ detected prominent and marginated nucleoli

Figure 3 (a and $\mathbf{b}$ ) Cords of atypical cells (a) and cribriform glands (b) represent uncommon patterns of invasion distinctive of highergrade prostate cancer.

in $25 \%$ and $7 \%$, respectively, of consecutive unselected benign prostate needle core biopsies; however, none of the benign specimens showed multiple nucleoli.

Although nuclear enlargement is not given the same diagnostic weight as prominent nucleoli, it is often a very helpful and easily applicable criterion. Nucleomegaly is commonly seen $(77-96 \%)$ in prostate cancer $^{4,5,21}$ and seems to correlate with Gleason score and with the presence of prominent nucleoli.

\section{Cytoplasmic features and intraluminal content}

The nature of the cytoplasm may be critical in the diagnosis of malignancy. At least in some cases, the cytoplasm of malignant prostate glands appears to be more amphophilic than the pale-to-clear cytoplasm of the surrounding or adjacent benign glands, provided the benign glands are appropriately stained $^{2,4,5}$ (Figure 6). 

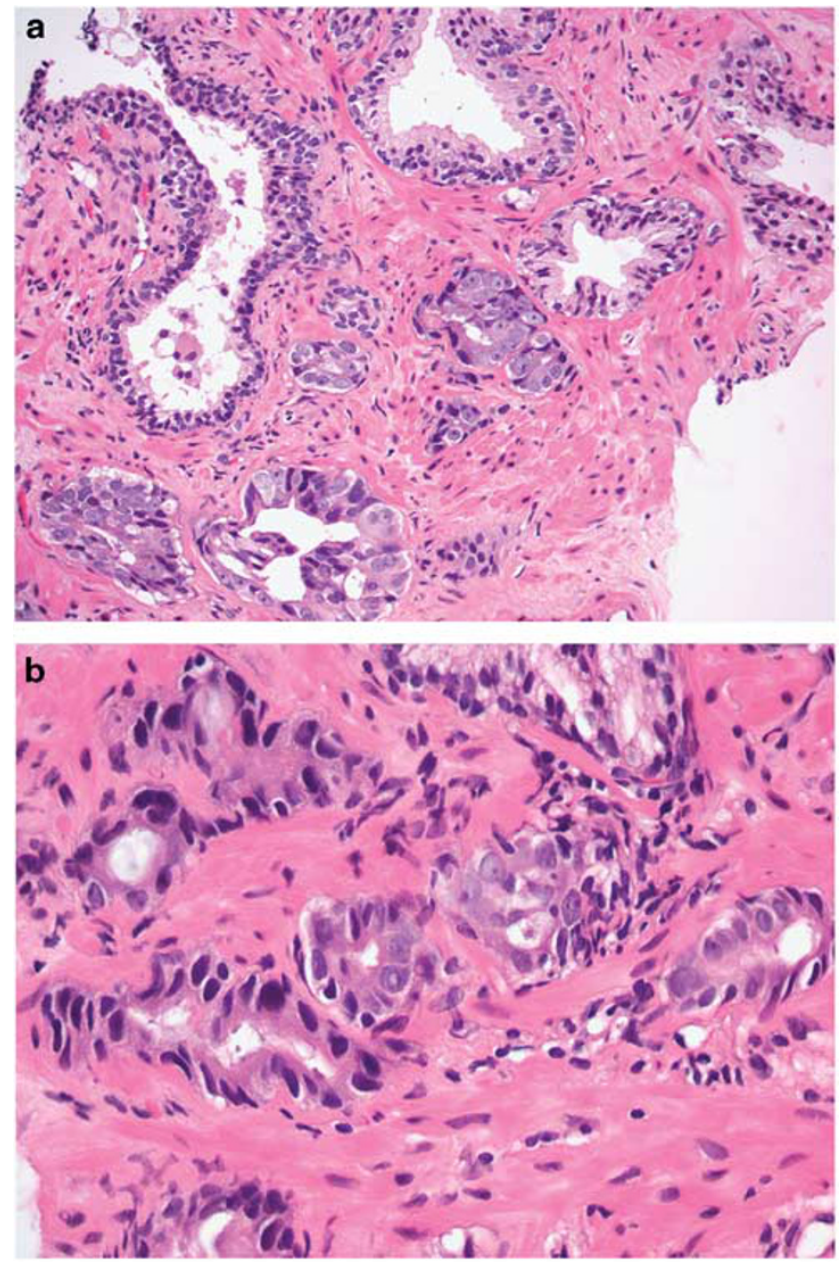

Figure 4 (a and b) Prostate cancer acini with prominent nuclear and nucleolar enlargement. Nuclear hyperchromatism (b) may also help in distinguishing malignant (left) from benign glands (right upper corner).

Intraluminal contents have received considerable interest as ancillary clues in the diagnosis of malignancy. Intraluminal amorphous eosinophilic material (Figure 7) is present in approximately 50$100 \%$ of cancer glands on needle biopsy ${ }^{4,5}$ and only occasionally in benign glands. ${ }^{15}$ Hard proteinaceous secretions are commonly noted in relation to crystalloids (Figure 8) and may represent their precursors. It is important to distinguish amorphous secretions from fractured corpora amylacea within benign glands; pink amorphous secretions lack the rounded, lamellar structure of corpora amylacea.

Blue-tinged intraluminal mucinous secretion (Figure 8) is a very useful criterion to assist in the diagnosis of limited prostate cancer. ${ }^{22}$ Although seen in up to half of prostate cancers on biopsy, luminal blue mucin appears to be more specific than pink secretions $^{4,7}$ and relatively sensitive. The prevalence of blue-tinged secretions is influenced by the nature of the H\&E stain. Acidic mucin may be present in benign mimickers, such as adenosis, sclerosis adenosis, basal cell hyperplasia and atrophic glands. ${ }^{23}$
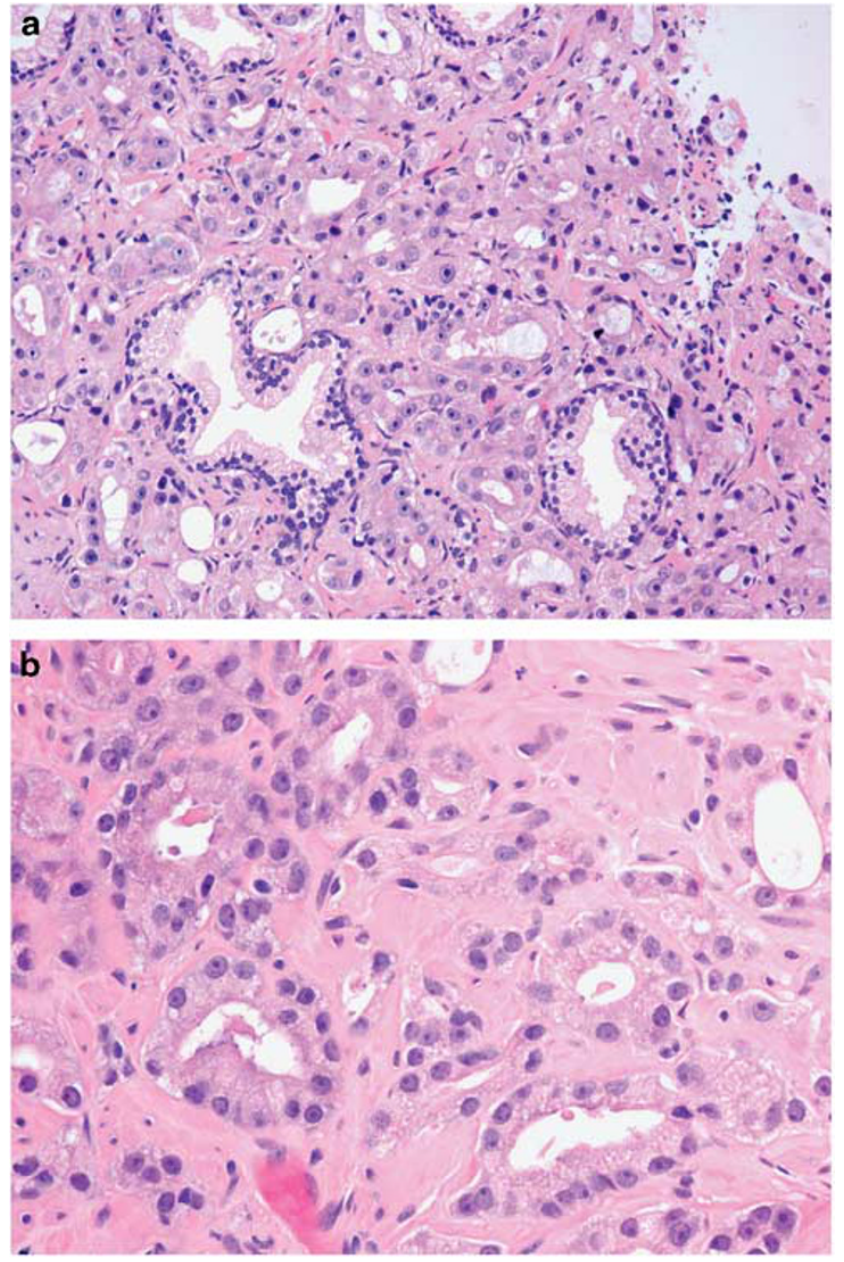

Figure 5 (a and b) Prominent nucleoli are detected to some extent in the majority of prostate cancer cases and have been considered one of the most important diagnostic features.

Varma et al ${ }^{15}$ failed to identify blue-tinged mucinous secretions in 100 benign prostate biopsy specimens but found intraluminal amorphous eosinophilic material in $2 \%$ of cases.

Crystalloids (Figure 9) are intraluminal dense needle-like eosinophilic structures with various geometric shapes, such as rectangular, hexagonal, triangular and rod-like structures. The reported incidence of intraluminal crystalloids varies from prostate needle biopsies $(10-41 \%)^{4,5,7,15}$ to cystoprostatectomy specimens $(60 \%){ }^{24,25}$ There seem to be an inverse correlation between the presence of crystalloids and Gleason score. ${ }^{4}$ As crystalloids have been found within the lumen of $1-5 \%$ of benign glands ${ }^{15,21,26}$ and are frequently seen in adenosis, ${ }^{2}$ it is important to carefully asses the morphology of the glands.

\section{Other minor criteria}

Although mitoses have been identified more often in limited foci of prostate cancer (up to $11 \%)^{4,7}$ 

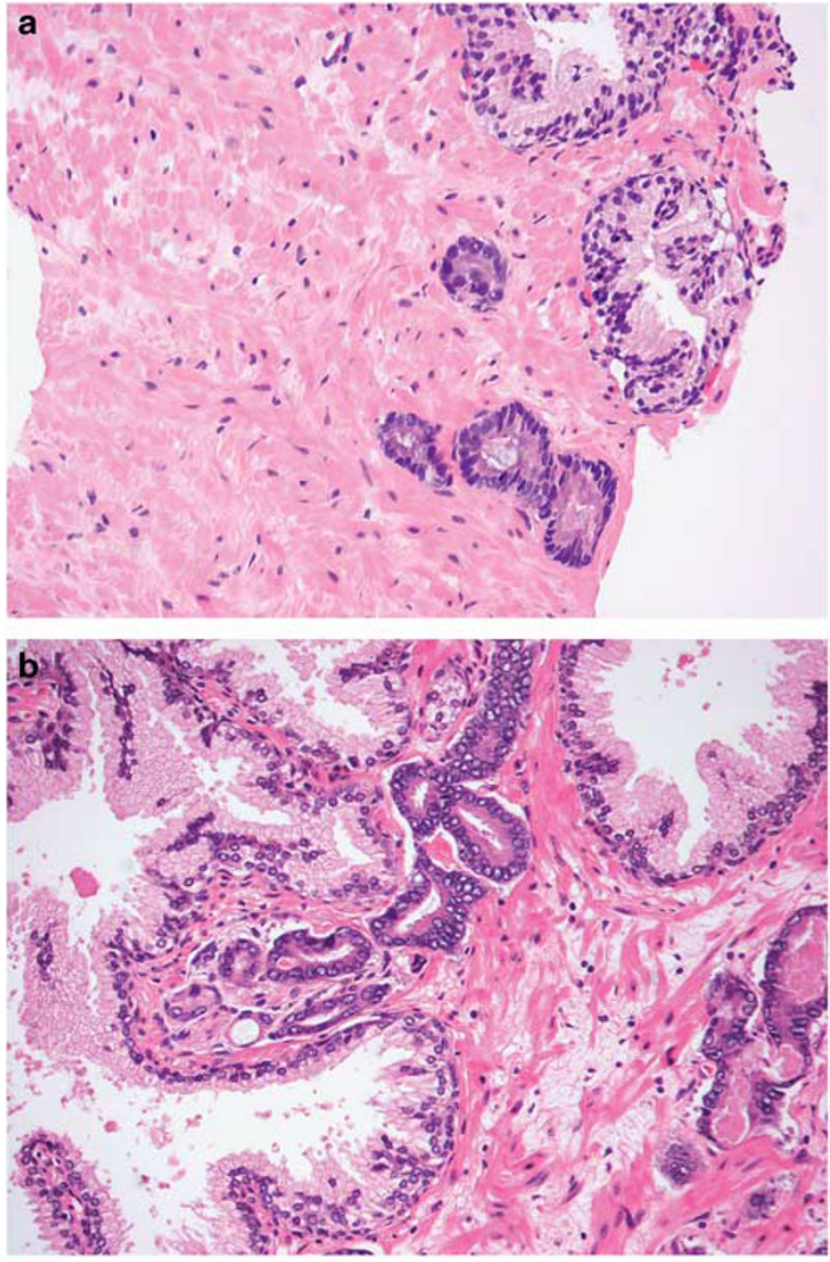

Figure 6 (a and $\mathbf{b}$ ) The cytoplasm of malignant prostate glands is more amphophilic than the pale-to-clear cytoplasm of the adjacent (a) or surrounding (b) benign glands.

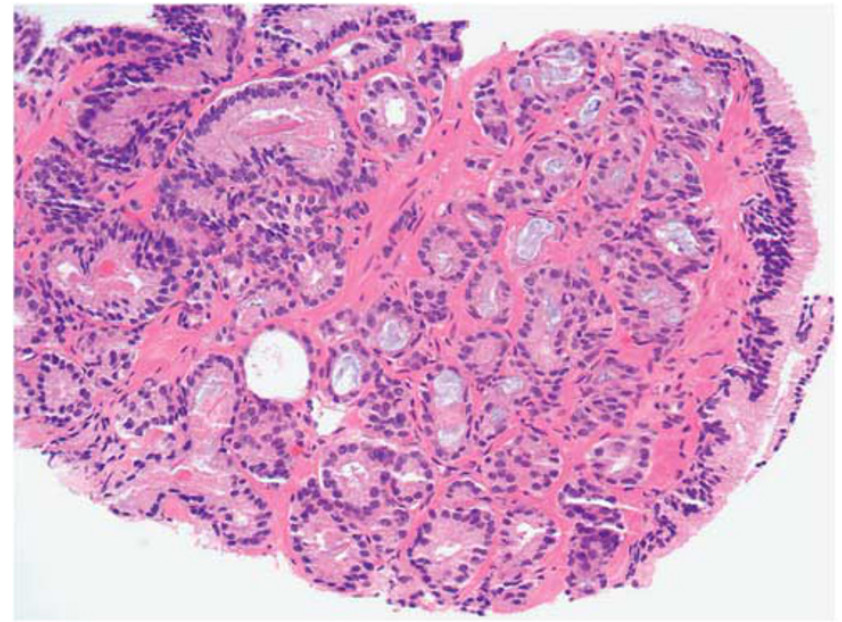

Figure 8 Prostate cancer glands with luminal blue mucin, pink amorphous secretions and crystalloids.

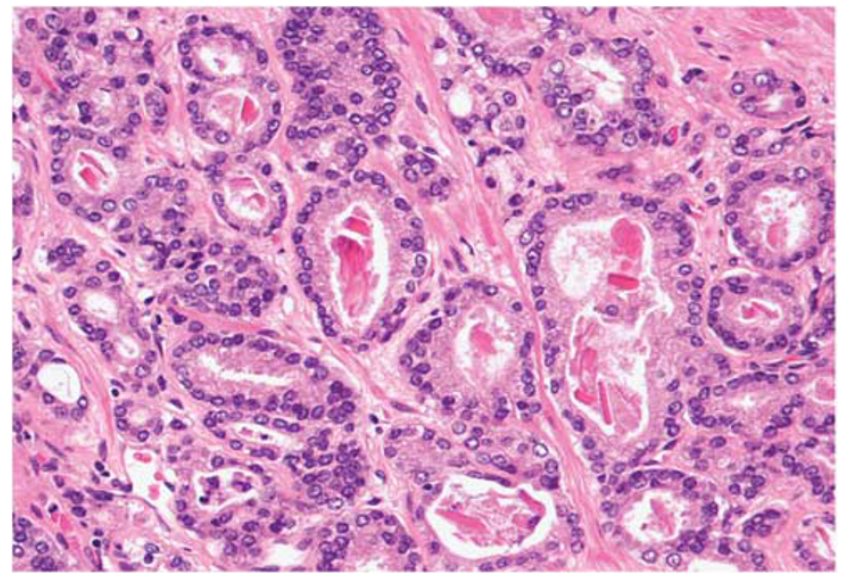

Figure 9 Prostate cancer glands with intraluminal crystalloids.

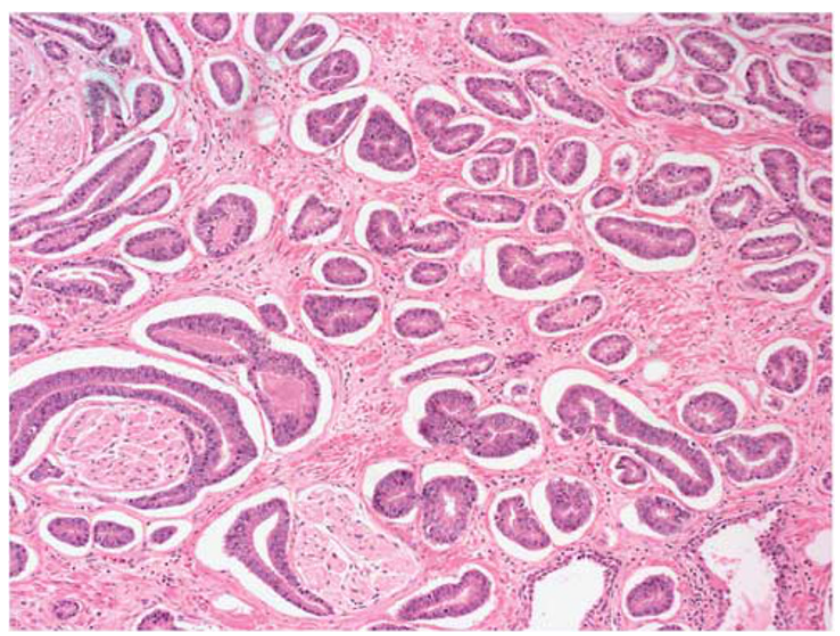

Figure 10 Prostate cancer with prominent periacinar retraction clefting. The glands seem to float freely within lacunar spaces. The clefts affect $>50 \%$ of the circumference in most of the glands. 

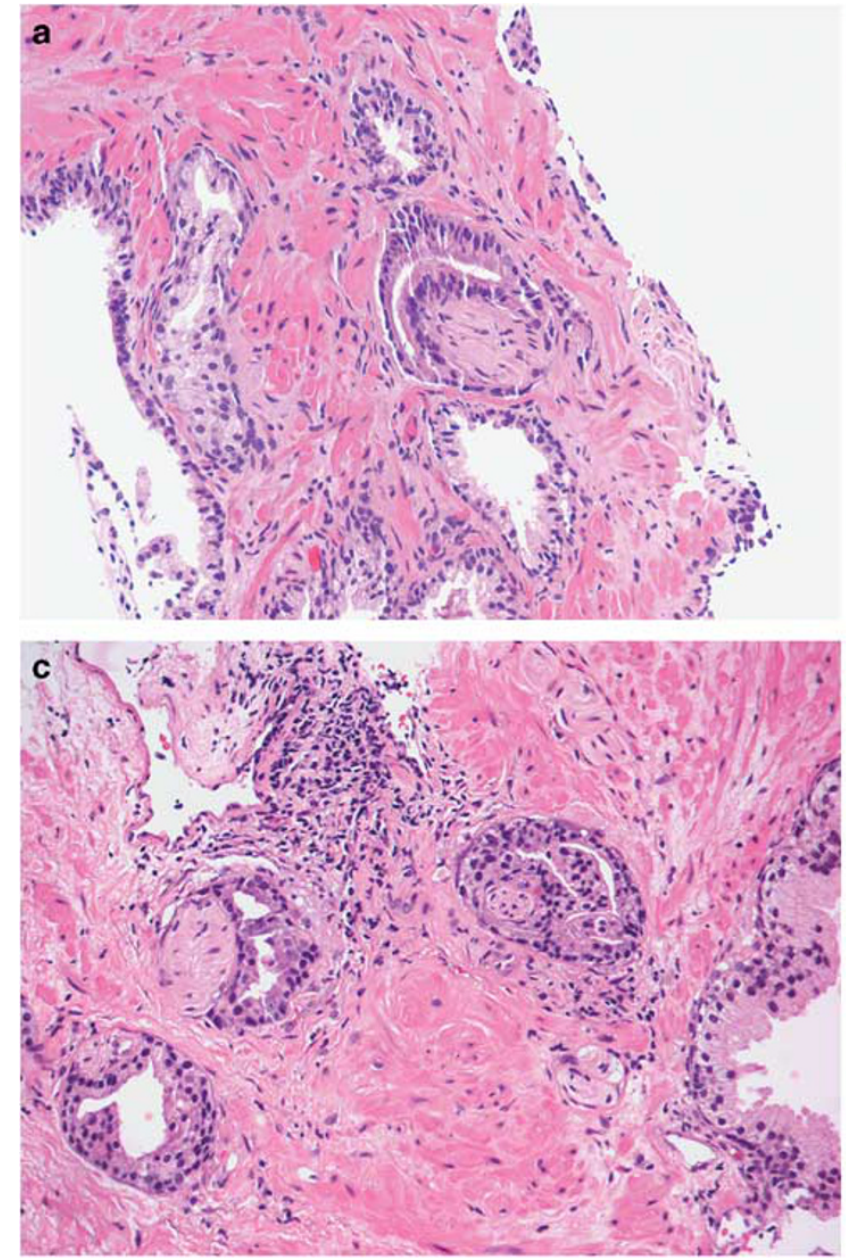
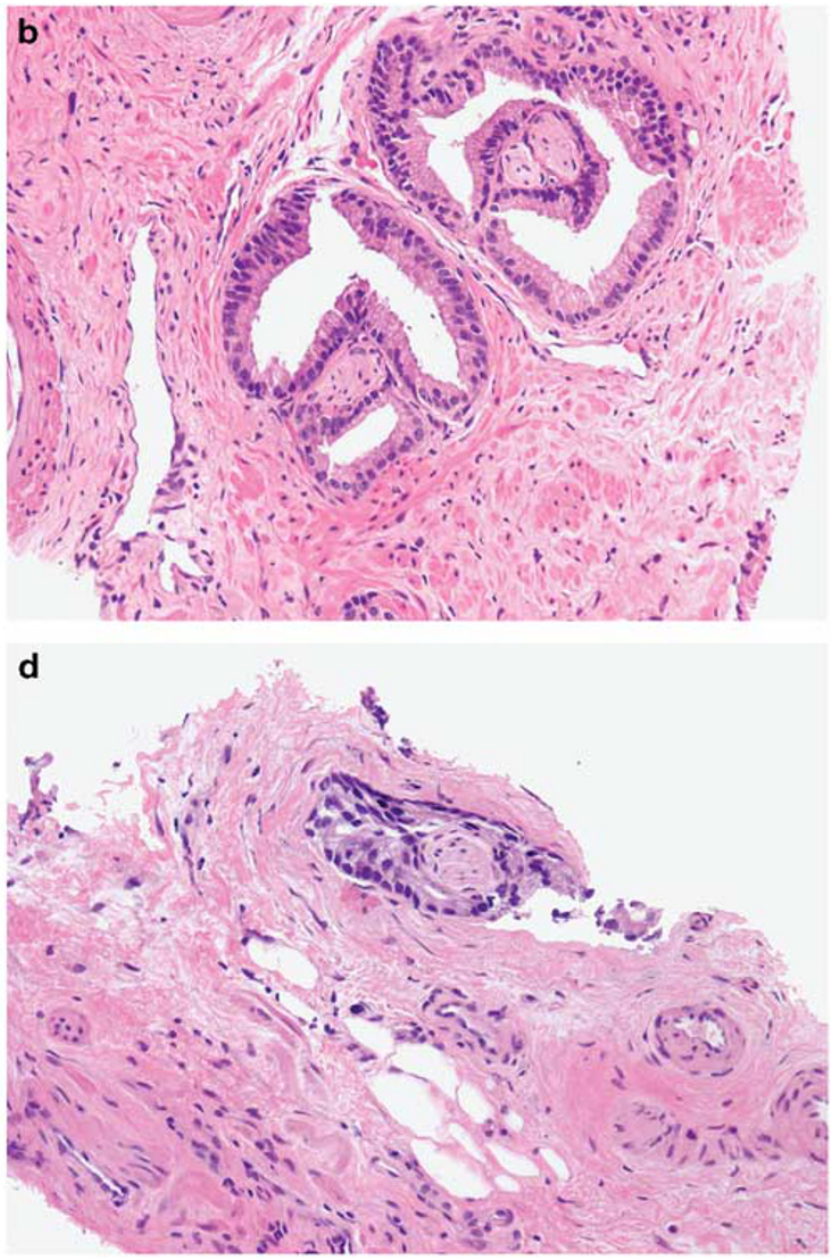

Figure 11 (a-d) Perineural invasion in limited prostate cancer. In order to use perineural invasion as a pathognomonic feature, the glands in question have to encircle the nerves (circumferential growth).

compared with atypical small acinar proliferations $(0 \%), 7$ their diagnostic helpfulness is limited as they are too rare to be a reliable finding. Close proximity to high-grade prostatic intraepithelial neoplasia has been reported as the least common $(13 \%)$ minor criterion in small foci of atypical glands. ${ }^{4}$

Periacinar retraction clefting (Figure 10) has been identified in approximately $40 \%$ of cancer biopsies vs $7 \%$ of benign biopsies. Extensive clefting is much more specific for cancer, but is rather infrequent. ${ }^{15,27}$ Periacinar retraction clefting represents a reliable criterion for the diagnosis of prostatic adenocarcinoma, particularly in cases with clefts affecting $>50 \%$ of the circumference in at least $50 \%$ of the suspicious glands. ${ }^{28}$

Corpora amylacea, atrophic glands, significant inflammation and glands merging with benign glands are features against the diagnosis of prostate cancer.

\section{Diagnostic features of prostate cancer}

Only few features are diagnostic in and of themselves for prostate cancer: perineural invasion, glomerulations, and mucinous fibroplasia (also known as collagenous micronodule).,6,29-31 These features are not detected in benign glands ${ }^{15}$ but are uncommon on needle biopsy specimens with minimal prostate cancer, limiting their diagnostic value.

Perineural invasion has been identified in 11-37\% of biopsies harboring prostate cancer, ${ }^{15}$ but is an uncommon finding $(0-3 \%)$ in limited prostate cancer. ${ }^{4,5,7,29}$ In order to use perineural invasion as a pathognomonic feature, the glands in question should encircle the nerve (circumferential growth) or show intraneural invasion (Figure 11). In view of the fact that benign glands can indent nerves, it is extremely important to distinguish perineural indentation from perineural invasion. Perineural indentation by benign glands is characterized by a lack of complete circumscription of the nerve.

Glomerulation consists of glands with a cribriform proliferation (tuft) attached to only one edge of the gland, resulting in a structure resembling a glomerulus (Figure 12). Although detected in $3-15 \%$ of tumors on needle biopsy, glomerulations are rarely seen in small foci of prostate cancer. ${ }^{15,29,31}$ 

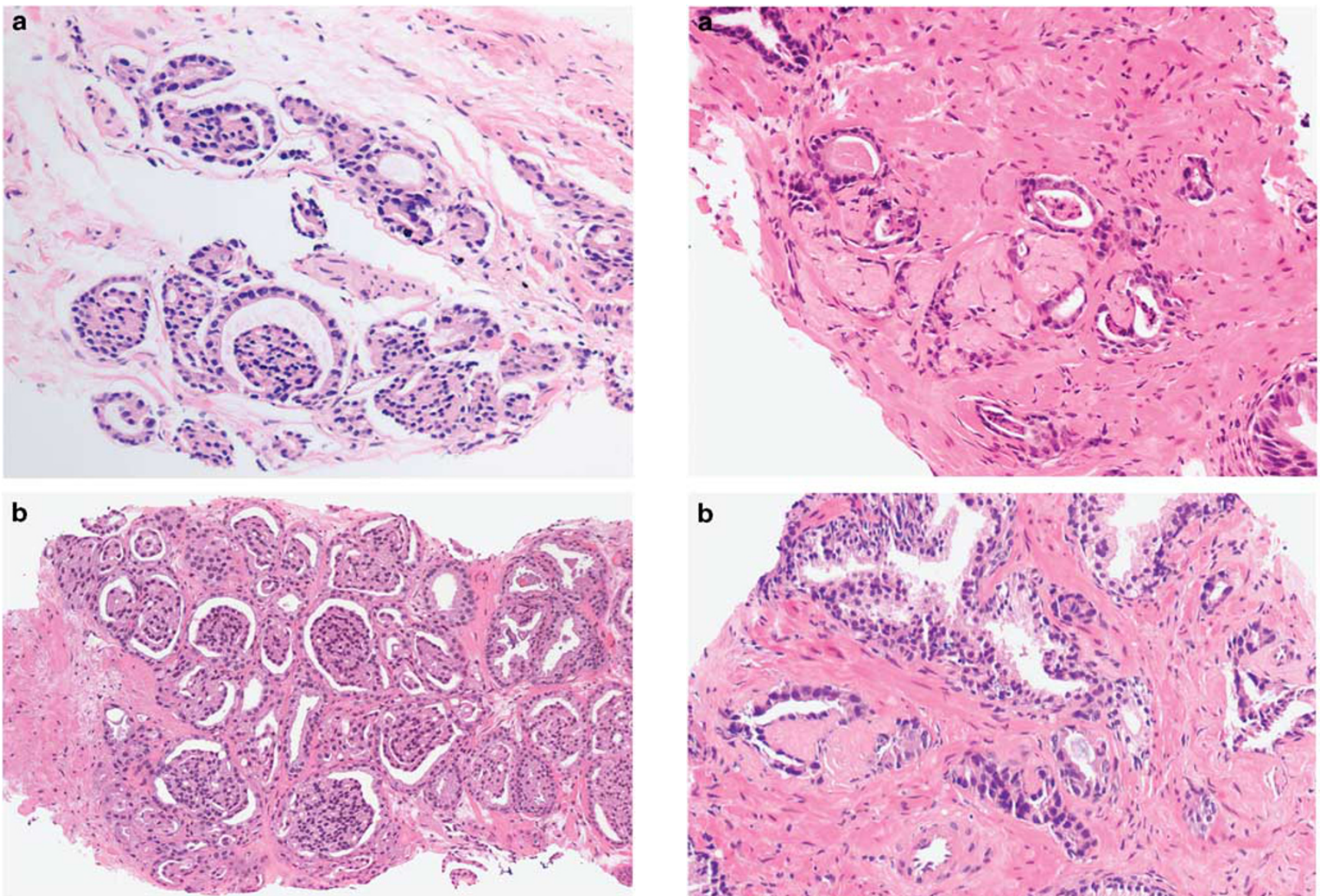

Figure 12 (a and b) Glomerulations consist of prostatic glands with a cribriform tuft attached to only one edge of the gland, resulting in a structure resembling a glomerulus.

Glomerulation has not been observed in benign prostate needle biopsies. ${ }^{15}$

Mucinous fibroplasia (or collagenous micronodule) is characterized by very delicate loose eosinophilic fibrous tissue with an ingrowth of fibroblasts that impinge on acinar lumens (Figure 13). ${ }^{30}$ Mucinous fibroplasia is a distinctive stromal response to invasive prostate cancer and has not been reported in benign or hyperplastic epithelium or in high-grade prostatic intraepithelial neoplasia. ${ }^{3,15}$ However, it is an infrequent (1-4\%) diagnostic finding in limited prostate cancer on needle biopsy. $5,7,15,29,30$

Seminal vesicle invasion and extraprostatic extension, although uncommon on needle biopsy, are diagnostic features of prostate cancer.

\section{Atypical small acinar proliferation}

The histological features that most often preclude the differentiation of prostatic adenocarcinoma from its benign mimickers include the small size of the focus in question (minimally sampled lesion), disappearance on deeper levels, lack of significant cytological atypia, biopsy and tissue processing artifact, atypical morphology seen in both malignant and benign
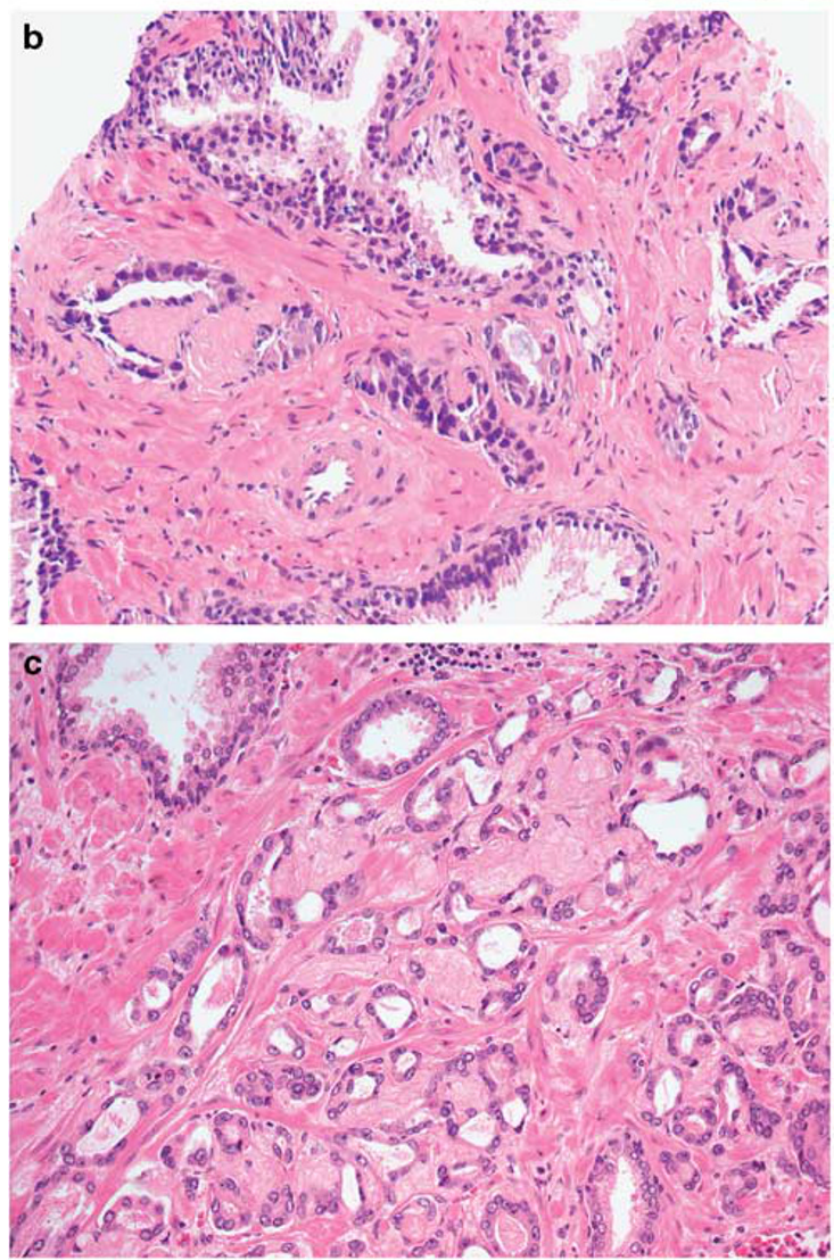

Figure 13 (a-c) Mucinous fibroplasia (or collagenous micronodule) is characterized by very delicate lose eosinophilic fibrous tissue with an ingrowth of fibroblasts that impinge on acinar lumens.

lesions, confusing immunohistochemical stains and associated inflammation. ${ }^{32}$

Lesions falling short of the threshold for the diagnosis of prostate cancer are diagnosed as ‘atypical small acinar proliferation' or 'small focus 


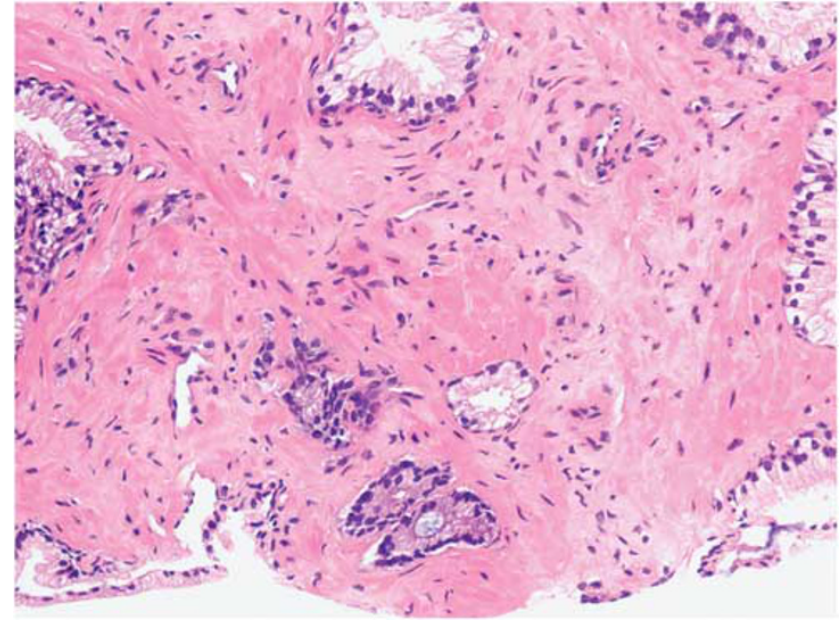

Figure 14 Prostate biopsy with atypical small acinar proliferation at the edge of the biopsy core. The atypical glands disappeared on deeper levels. Although highly suspicious, the findings are not diagnostic of prostatic adenocarcinoma. of atypical glands'33-35 (Figure 14). In such cases, repeat biopsy is recommended.

\section{Role of immunohistochemistry in the diagnosis of limited prostate cancer}

Although the light microscopic findings remain the gold standard for the diagnosis of prostatic carcinoma, difficult cases may benefit from immunohistochemical studies. The absence of basal cells, a major criteria we rely upon when making the diagnosis of prostate cancer, may be difficult to evaluate in routine tissue sections and may need to be confirmed by immunohistochemical markers, particularly when dealing with a limited number of atypical glands. The appearance of basal cells may vary substantially; tangentially cut secretory cells and stromal fibroblasts may mimic basal cells. ${ }^{15}$ Distorted and crushed tumor cells in small cancer foci can mimic basal cells.
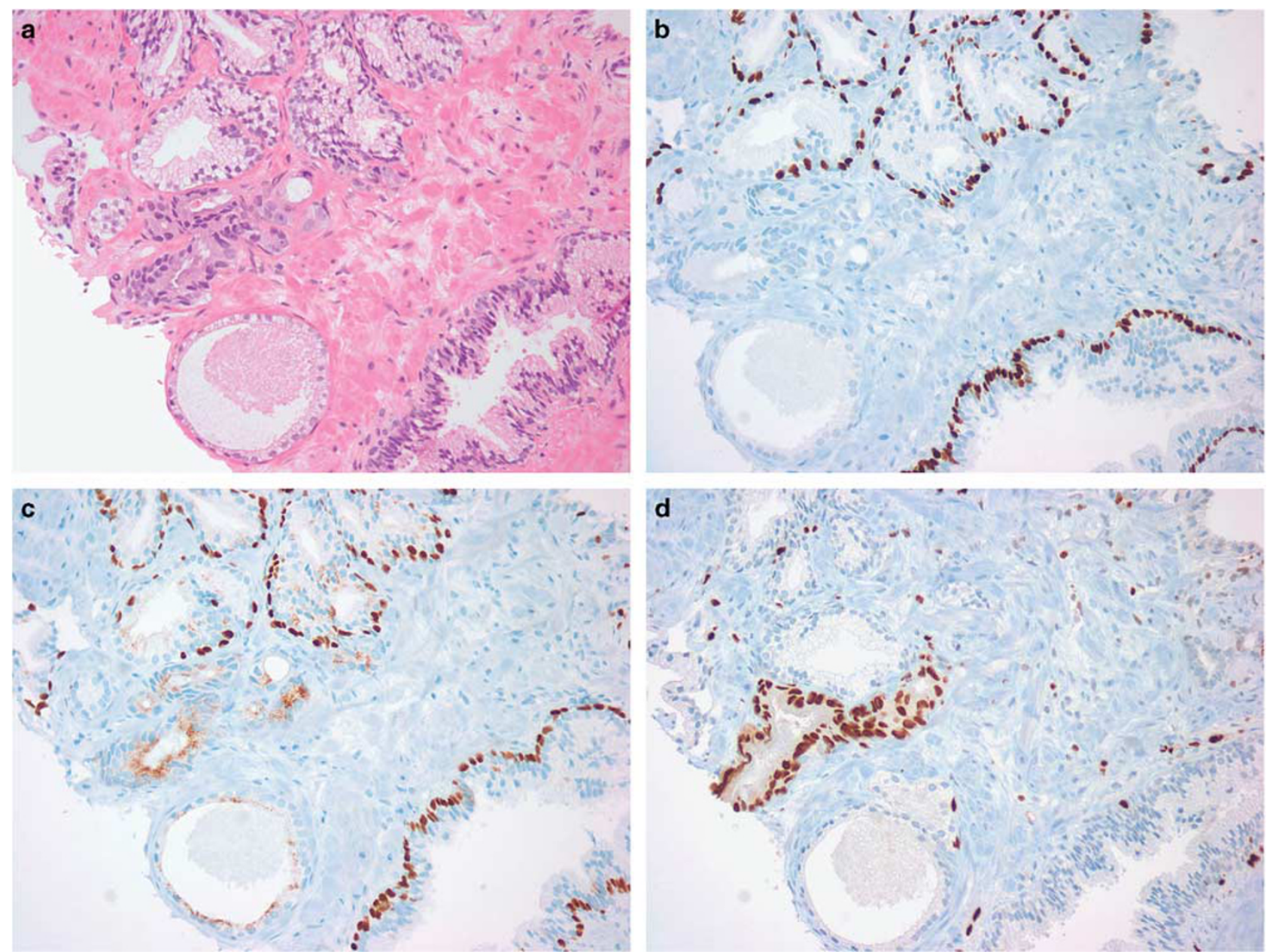

Figure 15 (a-d) Limited prostate cancer. In conjunction with the morphological features (a), negative p63 staining (b), AMACR expression (c) and strong ERG nuclear staining (d) help to establish a definitive diagnosis of limited prostate cancer. 
Although the use of basal cell-specific immunohistochemical stains (KER 903, p63, CK5/6) can be helpful in establishing a malignant diagnosis, it is important to keep in mind that a distinct layer of basal cells is not always present in benign small glands; consequently, the absence of basal cells in a small focus of atypical glands is not an absolute criterion of malignancy. ${ }^{2,13}$ P63 is more specific than other basal cell markers, ${ }^{36}$ although expressed in an aberrant manner in an unusual variant of prostate cancer. ${ }^{37-39}$ The diagnosis of prostate cancer with aberrant diffuse p63 expression is based on the morphology and confirmed by the absence of high molecular weight cytokeratin staining and positivity for a-methylacyl-CoA racemase (AMACR) in the atypical glands. False negative staining for basal cell markers may be seen in benign mimickers.

AMACR is commonly used in conjunction with morphology and basal cell markers to help establish a diagnosis of limited PCA ${ }^{40-43}$ (Figure 15). AMACR cytoplasmic staining is expressed by approximately $80 \%$ of limited prostatic adenocarcinoma on needle biopsy. Variants of prostate cancer more difficult to recognize, such as foamy, pseudohyperplastic and atrophic, are labeled with AMACR in only $60-70 \%$ of cases., ${ }^{44,45}$ In addition, AMACR is not entirely specific for adenocarcinoma, as it stains most cases of high-grade prostatic intraepithelial neoplasia, ${ }^{46}$ some foci of adenosis ${ }^{47}$ and occasionally benign glands. ${ }^{48}$

The use of double staining or triple staining has the advantage to conserve tissue. ${ }^{49}$ ERG, although highly specific, is not very sensitive and expressed in only $40-60 \%$ of prostate cancer cases. ${ }^{49-52}$

\section{Conclusions}

When handling prostate needle biopsies with limited atypical glands suspicious for prostatic adenocarcinoma, the best approach is to first evaluate the benign glands for reference and the atypical glands for cytological and architectural atypia. The diagnosis of prostate cancer relies on a constellation of features. The most useful diagnostic criteria are infiltrative growth pattern, best evaluated at low-tomedium magnification, and prominent nucleoli and lack of basal cells, best appreciated at high magnification. When both cytological and architectural atypia are present, after excluding potential benign conditions that may cause atypia, a diagnosis of prostate cancer can be made with confidence. Correlation of morphological features with adjunctive immunohistochemical stains may be necessary to make the correct diagnosis, particularly when dealing with limited foci of atypical glands.

If satisfying architectural and cytological diagnostic criteria of prostate cancer are lacking, one should consider a diagnosis of atypical glands suspicious for cancer. Additional sections and/or special stains may help in the evaluation of difficult cases.

\section{Disclosure/conflict of interest}

The author declares no conflict of interest.

\section{References}

1 Thorson P, Humphrey PA. Minimal adenocarcinoma in prostate needle biopsy tissue. Am J Clin Pathol 2000;114:896-909.

2 Epstein JI. Diagnosis and reporting of limited adenocarcinoma of the prostate on needle biopsy. Mod Pathol 2004;17:307-315.

3 Bostwick DG, Iczkowski KA. Minimal criteria for the diagnosis of prostate cancer on needle biopsy. Ann Diagn Pathol 1997;1:104-129.

4 Epstein JI. Diagnostic criteria of limited adenocarcinoma of the prostate on needle biopsy. Hum Pathol 1995;26:223-229.

5 Leroy X, Aubert S, Villers A, et al. Minimal focus of adenocarcinoma on prostate biopsy: clinicopathological correlations. J Clin Pathol 2003;56:230-232.

6 Algaba F, Epstein JI, Aldape HC, et al. Assessment of prostate carcinoma in core needle biopsy-definition of minimal criteria for the diagnosis of cancer in biopsy material. Cancer 1996;78:376-381.

7 Iczkowski KA, Bostwick DG. Criteria for biopsy diagnosis of minimal volume prostatic adenocarcinoma: analytic comparison with nondiagnostic but suspicious atypical small acinar proliferation. Arch Pathol Lab Med 2000;124:98-107.

8 Thorson P, Vollmer RT, Arcangeli C, et al. Minimal carcinoma in prostate needle biopsy specimens: diagnostic features and radical prostatectomy follow-up. Mod Pathol 1998;11:543-551.

9 Kelemen PR, Buschmann RJ, Weisz-Carrington P. Nucleolar prominence as a diagnostic variable in prostatic carcinoma. Cancer 1990;65:1017-1020.

10 Kovi J. Microscopic differential diagnosis of small acinar adenocarcinoma of prostate. Pathol Annu 1985;20(Pt 1):157-196.

11 Mostofi FK, Sesterhenn IA, Davis CJ Jr. Prostatic carcinoma: problems in the interpretation of prostatic biopsies. Hum Pathol 1992;23:223-241.

12 Srigley JR. Small-acinar patterns in the prostate gland with emphasis on atypical adenomatous hyperplasia and smallacinar carcinoma. Semin Diagn Pathol 1988;5:254-272.

13 Totten RS, Heinemann MW, Hudson PB, et al. Microscopic differential diagnosis of latent carcinoma of prostate. AMA Arch Pathol 1953;55:131-141.

14 Kramer CE, Epstein JI. Nucleoli in low-grade prostate adenocarcinoma and adenosis. Hum Pathol 1993;24: 618-623.

15 Varma M, Lee MW, Tamboli P, et al. Morphologic criteria for the diagnosis of prostatic adenocarcinoma in needle biopsy specimens. A study of 250 consecutive cases in a routine surgical pathology practice. Arch Pathol Lab Med 2002;126:554-561.

16 Helpap B. Observations on the number, size and localization of nucleoli in hyperplastic and neoplastic prostatic disease. Histopathology 1988;13:203-211.

17 Nelson RS, Epstein JI. Prostatic carcinoma with abundant xanthomatous cytoplasm. Foamy gland carcinoma. Am J Surg Pathol 1996;20:419-426.

18 Epstein JI, Grignon DJ, Humphrey PA, et al. Interobserver reproducibility in the diagnosis of prostatic intraepithelial neoplasia. Am J Surg Pathol 1995;19:873-886. 
19 Egevad L, Allsbrook WC, Epstein JI. Current practice of diagnosis and reporting of prostatic intraepithelial neoplasia and glandular atypia among genitourinary pathologists. Mod Pathol 2006;19:180-185.

20 Epstein JI, Armas OA. Atypical basal cell hyperplasia of the prostate. Am J Surg Pathol 1992;16:1205-1214.

21 Henneberry JM, Kahane H, Humphrey PA, et al. The significance of intraluminal crystalloids in benign prostatic glands on needle biopsy. Am J Surg Pathol 1997;21:725-728.

22 Epstein JI, Fynheer J. Acidic mucin in the prostate: can it differentiate adenosis from adenocarcinoma? Hum Pathol 1992;23:1321-1325.

23 Gaudin PB, Epstein JI. Adenosis of the prostate. Histologic features in needle biopsy specimens. Am J Surg Pathol 1995;19:737-747.

24 Furusato M, Kato H, Takahashi $\mathrm{H}$, et al. Crystalloids in latent prostatic carcinoma. Prostate 1989;15:259-262.

25 Ro JY, Grignon DJ, Troncoso P, et al. Intraluminal crystalloids in whole-organ sections of prostate. Prostate 1988;13:233-239.

26 Anton RC, Chakraborty S, Wheeler TM. The significance of intraluminal prostatic crystalloids in benign needle biopsies. Am J Surg Pathol 1998;22:446-449.

27 Ulamec M, Tomas D, Ensinger C, et al. Periacinar retraction clefting in proliferative prostatic atrophy and prostatic adenocarcinoma. J Clin Pathol 2007;60:1098-1101.

28 Kruslin B, Tomas D, Rogatsch $\mathrm{H}$, et al. Periacinar retraction clefting in the prostatic needle core biopsies: an important diagnostic criterion or a simple artifact? Virchows Arch 2003;443:524-527.

29 Baisden BL, Kahane H, Epstein JI. Perineural invasion, mucinous fibroplasia, and glomerulations: diagnostic features of limited cancer on prostate needle biopsy. Am J Surg Pathol 1999;23:918-924.

30 Bostwick DG, Wollan P, Adlakha K. Collagenous micronodules in prostate cancer. A specific but infrequent diagnostic finding. Arch Pathol Lab Med 1995;119:444-447.

31 Pacelli A, Lopez-Beltran A, Egan AJ, et al. Prostatic adenocarcinoma with glomeruloid features. Hum Pathol 1998;29:543-546.

32 Shah RB, Zhou M. Prostate Biopsy Interpretation: An Illustrated Guide. Springer: Berlin, Heidelberg, Germany, 2012.

33 Iczkowski KA, MacLennan GT, Bostwick DG. Atypical small acinar proliferation suspicious for malignancy in prostate needle biopsies: clinical significance in 33 cases. Am J Surg Pathol 1997;21:1489-1495.

34 Cheville JC, Reznicek MJ, Bostwick DG. The focus of "atypical glands, suspicious for malignancy" in prostatic needle biopsy specimens: incidence, histologic features, and clinical follow-up of cases diagnosed in a community practice. Am J Clin Pathol 1997;108:633-640.

35 Epstein JI. Atypical small acinar proliferation of the prostate gland. Am J Surg Pathol 1998;22:1430-1431.

36 Ali TZ, Epstein JI False. positive labeling of prostate cancer with high molecular weight cytokeratin: p63 a more specific immunomarker for basal cells. Am J Surg Pathol 2008;32:1890-1895.

37 Giannico GA, Ross HM, Lotan T, et al. Aberrant expression of p63 in adenocarcinoma of the prostate: a radical prostatectomy study. Am J Surg Pathol 2013;37:1401-1406.
38 Osunkoya AO, Hansel DE, Sun X, et al. Aberrant diffuse expression of p63 in adenocarcinoma of the prostate on needle biopsy and radical prostatectomy: report of 21 cases. Am J Surg Pathol 2008;32: 461-467.

$39 \mathrm{Wu}$ A, Kunju LP. Prostate cancer with aberrant diffuse p63 expression: report of a case and review of the literature and morphologic mimics. Arch Pathol Lab Med 2013;137:1179-1184.

40 Magi-Galluzzi C, Luo J, Isaacs WB, et al. Alphamethylacyl-CoA racemase: a variably sensitive immunohistochemical marker for the diagnosis of small prostate cancer foci on needle biopsy. Am J Surg Pathol 2003;27:1128-1133.

41 Zhou M, Aydin H, Kanane H, et al. How often does alpha-methylacyl-CoA-racemase contribute to resolving an atypical diagnosis on prostate needle biopsy beyond that provided by basal cell markers? Am J Surg Pathol 2004;28:239-243.

42 Trpkov K, Bartczak-McKay J, Yilmaz A. Usefulness of cytokeratin 5/6 and AMACR applied as double sequential immunostains for diagnostic assessment of problematic prostate specimens. Am J Clin Pathol 2009;132: 211-220; quiz 307.

43 Hameed O, Humphrey PA. Immunohistochemistry in diagnostic surgical pathology of the prostate. Semin Diagn Pathol 2005;22:88-104.

44 Farinola MA, Epstein JI. Utility of immunohistochemistry for alpha-methylacyl-CoA racemase in distinguishing atrophic prostate cancer from benign atrophy. Hum Pathol 2004;35:1272-1278.

45 Zhou M, Jiang Z, Epstein JI. Expression and diagnostic utility of alpha-methylacyl-CoA-racemase (P504S) in foamy gland and pseudohyperplastic prostate cancer. Am J Surg Pathol 2003;27:772-778.

$46 \mathrm{Wu}$ CL, Yang XJ, Tretiakova M, et al. Analysis of alphamethylacyl-CoA racemase (P504S) expression in highgrade prostatic intraepithelial neoplasia. Hum Pathol 2004;35:1008-1013.

47 Armah HB, Parwani AV. Atypical adenomatous hyperplasia (adenosis) of the prostate: a case report with review of the literature. Diagn Pathol 2008;3:34

48 Worschech A, Meirelles L, Billis A. Expression of alpha-methylacyl coenzyme A racemase in partial and complete focal atrophy on prostate needle biopsies. Anal Quant Cytol Histol 2009;31:424-431.

49 Epstein JI, Egevad L, Humphrey PA, et al. Members of the IIIDUPG. Best practices recommendations in the application of immunohistochemistry in the prostate: report from the International Society of Urologic Pathology consensus conference. Am J Surg Pathol 2014;38:e6-e19.

50 Yaskiv O, Rubin BP, He H, et al. ERG protein expression in human tumors detected with a rabbit monoclonal antibody. Am J Clin Pathol 2012;138:803-810.

51 Andrews C, Humphrey PA. Utility of ERG versus AMACR expression in diagnosis of minimal adenocarcinoma of the prostate in needle biopsy tissue. Am J Surg Pathol 2014;38:1007-1012.

52 Lee SL, Yu D, Wang C, et al. ERG Expression in Prostate Needle Biopsy: Potential Diagnostic and Prognostic Implications. Appl Immunohistochem Mol Morphol 2015;23:499-505. 